\title{
Risk factors for placental malaria and associated adverse pregnancy outcomes in Rufiji, Tanzania: a hospital based cross sectional study.
}

\author{
Rabi Ndeserua ${ }^{1}$, Adinan Juma ${ }^{2}$, Dominic Mosha ${ }^{3}$, Jaffu Chilongola ${ }^{4}$
}

1. Muhimbili Orthopaedic Institute.

2. Kilimanjaro Christian Medical Center, Community Health Department.

3. Kilimanjaro Christian Medical University College, Community Health Department

4. Kilimanjaro Christian Medical University College, Biochemistry and Mol Biology

\begin{abstract}
Background: Prevention and treatment of malaria during pregnancy is crucial for reduction of malaria in pregnancy and its adverse outcomes. The spread of parasite resistance to Sulphadoxine-Pyrimethamine (SP) used for Intermittent Preventive Treatment for malaria in pregnancy (IPTp), particularly in East Africa has raised concerns about the usefulness and the reliability of the IPTp regimen. We aimed to assess the effectiveness of two doses of SP in treating and preventing occurrence of adverse pregnancy outcomes.

Methodology: The study was an analytical cross sectional study which enrolled 350 pregnant women from Kibiti Health Centre, South Eastern Tanzania. Structured questionnaires were used to obtain previous obstetrics and medical history of participants and verified by reviewing antenatal clinic cards. Maternal placental blood samples for microscopic examination of malaria parasites were collected after delivery. Data was analyzed for associations between SP dosage, risk for PM and pregnancy outcome. Sample size was estimated based on precision

Results: Prevalence of placental maternal (PM) was 8\% among pregnant women (95\%CI, 4.4-13.1\%). Factors associated with increased risk of $\mathrm{PM}$ were primigravidity $(\mathrm{P}<0.001)$ and history of fever during pregnancy $(\mathrm{P}=0.02)$. Use of at least 2 doses of SP for IPTp during pregnancy was insignificantly associated with reducing the risk $\mathrm{PM}(\mathrm{P}=0.08)$, low birth weight $(\mathrm{P}=0.73)$ and maternal anemia $(\mathrm{P}=0.71)$ but associated significantly with reducing the risk of preterm birth $(\mathrm{P}<0.001)$.

Conclusion: Two doses of SP for IPTp regime are ineffective in preventing and treating PM and adverse pregnancy outcome. Hence a review to the current IPTp regimen should be considered with possibility of integrating it with other malaria control strategies.
\end{abstract}

Keywords: Placental malaria, intermittent preventive treatment, Sulphadoxine-Pyrimethamine, Malaria in pregnancy

DOI: http://dx.doi.org/10.4314/ahs.v15i3.15

Cite as: Ndeserua R, Juma A, Mosha D, Chilongola J. Risk factors for placental malaria and associated adverse pregnancy outcomes in Rufji, Tanzania: a hospital based cross sectional study. Afri Health Sci. 2015;15(3):810-8. doi: http:// dx.doi.org/10.4314/ abs.v15i3.15

\section{Introduction}

Despite integrated efforts to control malaria in endemic areas, PM remains a significant cause of maternal and infant mortality and morbidity. Malaria in pregnancy remains a major public health problem especially in sub-Saharan African countries where approximately 25 million pregnant women are at risk of $P$. falciparum infection every year ${ }^{7}$. About $25 \%$ of these pregnant

\section{Corresponding author: \\ Jaffu Chilongola \\ Kilimanjaro Christian Medical \\ University College, Biochemistry \\ and Mol Biolog \\ Email: jchilx@yahoo.co.uk/ \\ j.chilongola@kcri.ac.tz}

women have evidence of placental parasitaemia during delivery and have increased risk of maternal and perinatal mortality ${ }^{8}$. In Tanzania, the prevalence of PM is $8 \%$ which is lower compared to the overall estimated prevalence for Africa ${ }^{19}$.

Available literature ascertains that, the parity pattern of malaria susceptibility in highly endemic areas show that primigravidae and secundigravidae (to a lesser extent) are more affected than multigravida ${ }^{22}$. P.falciparum parasites are known to invade the placenta in semi-immune women to cause PM, a condition associated with stillbirth, spontaneous abortion, fetal pre-maturity, and maternal anemia ${ }^{15}$, low birth weight (LBW). Most of these pregnancy outcomes are important risk factors for neonatal mortality and retarded growth regardless of the factors that affect malaria epidemiology in a given location $^{5,15,22,23,29}$. Many studies suggest that, multigravida 
women have less risk of PM than primigravida because they have developed immunity to sequestered parasites 10,11. In sub-Saharan Africa, 20\% of LBW has been attributed to malaria in pregnancy ${ }^{20}$.

Prevention and treatment of malaria during pregnancy is crucial for reduction of parasitaemia and improving birth outcomes. World Health Organization (WHO 2004) and international Roll BackMalaria (RBM) initiative recommended three cost effective protective measures for all pregnant women, which are the use of IPTp, insecticide-treated mosquito nets and effective case management of malarial illnesses ${ }^{40}$. In Tanzania, these recommendations are implemented through the National Malaria Control Program (NMCP) and up to $2010,57 \%$ of all pregnant women in Tanzania slept under an Insecticide Treated bed-Net (ITN) while 60\% of them used at least one dose of IPTp. Sulphadoxine-Pyrimethamine (SP) has been widely used for intermittent preventive treatment during pregnancy (IPTp) as a means to avoid poor outcomes that are associated with $\mathrm{PM}^{4}$.

IPTp using SP is recommended in areas of high malaria transmission with low resistance to SP (World Health Organization 2003). In Tanzania, the policy is to offer SP to all pregnant women attending antenatal clinics ${ }^{38}$ at between 20 and 24 weeks gestation for the first dose and between 28 and 32 weeks for the second dose ${ }^{2}$. The newly proposed regimen that requires administration of monthly IPTp dose from second trimester until delivery has not yet beeneffectively implemented in the country. It has been documented that, the proportion of pregnant women receiving single dose of SP in Zambia is about twice $(60 \%)$ as much as those receiving two doses $(27 \%)$, where single dose proved to be inferior to higher doses ${ }^{14,16}$.

The changing patterns in malaria epidemiology and pathogenesis may imply major shifts in traditional paradigms of associations between placental malaria and its risk factors on one hand, and its fetal-maternal outcomes on the other hand. We aimed at assessing the prevalence of placental malaria in women living in a high transmission setting, and to determine the risk factors associated with it, including Bednet use and SP prophylaxis as IPTp during pregnancy, and the effects on pregnancy outcomes.

\section{Methods}

\section{Study design and area}

This study was a hospital based descriptive cross sec- tional study involving pregnant women, conducted between September and December 2012 in Rufiji district, South-Eastern Tanzania. This is an area of high malaria transmission with prevalence of $20.8 \%$ (NBS, 2008). It lies 500 meters below sea level and receives an annual rainfall of $800-1000 \mathrm{~mm}$. According to data on malaria incidence from health care facilities, malaria is the most important public health problem for both adult and children in Rufiji District (Goodman et al. 2004). The most common occupation in the study area is subsistence farming, with rice, maize and cassava being the predominant food crops. Residents of the study area are also involved in subsistence animal husbandry, fishing and small-scale trading. Rufiji has very limited tarmac roads, a situation that at times of long rains isolates it from major highways and market centers ${ }^{27,28}$. Malaria transmission in this region, measured by the mean number of infected bites per person per year (entomological inoculation rate) has been estimated at between 79 and 1,209 infective bites per person per year, and malaria is the leading cause of outpatient diagnoses, and a high proportion of reported inpatient deaths. Pregnant women who attended Kibiti Health Centre (KHC) for delivery were recruited for the study.

\section{Sample size}

A minimum sample size of 136 women was estimated to provide a power of $80 \%$ with $5 \%$ precision. A total of 350 pregnant women with study's inclusion criteria were consecutively recruited from KHC which increased both the power and precision.

\section{Data collection}

Data was collected using a structured questionnaire by interviewing participating mothers in the labor ward. This involved mothers who were waiting to enter labor and the ones who are in latent phase of labor. The study's inclusion criteria were women who delivered in the study facility, availability of a placenta for pathological examination and mothers who agreed to participate in the study by providing written consent. Women with serious delivery complications including severe post-partum bleeding, eclampsia and other condition that lead to case referral to a tertiary hospital were excluded. Information regarding biographical, obstetrical and medical history of the participant during pregnancy was recorded. Information on the new born was also recorded including infant birth weight. Gestational length was estimated based on the date of recorded last normal menstrual period (LNMP) or fundal height examination, when LNMP was unknown. Newborns were assessed at delivery. All information of the partici- 
pant from antenatal clinic card and medical registry was careful reviewed by the principal investigator. This information included history of SP use, birth weights of infants and gravidity as well as the use of medications other than SP. Filling of all questions in the questionnaire was completed on the day of interview. Questionnaires completed by study assistants were reviewed in an evening roundup meeting to verify completeness and reliability of filled information. To determine placental malaria (parasitemia), placental blood samples were collected from the maternal side of the placenta by making an incision about $1.5 \mathrm{~cm}$ deep on the central maternal side by the pool-biopsy methods ${ }^{34}$ and hence, about $1 \mathrm{cc}$ of blood sample was collected from a small pool created in this incision after squeezing. Collected blood samples were used to prepare thick and thin blood smears on microscopic glass slides. Prepared smears were then stained with 5\% Giemsa's stain for 30 minutes, fixed in absolute methanol for 5 seconds, dried for $>45$ minutes and examined by two independent microscopists at 100X magnification guided by standard operating procedures (SOPs). Up to 100 High power fields (HPFs) in cases of very scanty malaria on a slide may be read and number of parasites counted against the white cells counted per HPF is recorded. A slide negative after 100 HPFs was read by two microscopists and declared negative. Discrepancy findings were reviewed by a third independent miscroscopist and results obtained were regarded as decisive. Hemoglobin was measured by using Abbott Cell-Dyn 3700 Hematology Analyzer in which a hemoglobin reading below $10 \mathrm{~g} / \mathrm{dL}$ was considered anemia. In addition a packed cell volume measured by spinning heparinized portion of the placental blood sample (PCV) of $30 \%$ was interpreted as anemia.

\section{Primary end points}

The primary endpoint of the study was placenta malaria. Secondary outcomes that were assessed under the study include preterm birth, low birth weight and maternal anaemia. Preterm birth was defined as birth before 37 weeks of gestational age. Low birth weight was defined as a birth weight below $2500 \mathrm{~g}$, and maternal anaemia was defined as haemoglobin level below $11 \mathrm{~g} /$ $\mathrm{dl}$ at the time of labor.

\section{Statistical analysis}

Data was analyzed using statistical package for social sciences (SPSS) version 18. Numerical variables were summarized into percentage. Categorical variables were summarized using cross-tabulation to estimate different proportion. The primary outcome was the proportion of women with placenta malaria. The bi-variate models include IPTp, maternal age, gravidity, history of fever in pregnancy, HIV status and ITNs use as explanatory variables. Multivariate analyses were performed using multiple logistic regressions to assess the effects of multiple factors on study's outcome and for controlling of confounders. Differences were considered to be significant at a cutoff point of $\mathrm{p} \leq 0.05$.

\section{Ethics}

Prior to enrollment, participants were informed on the objectives, procedures and benefits of the study. They were assured on anonymity and confidentiality throughout the study. Written consent was obtained before the participants were enrolled into the study. Approval for this study was obtained from Kilimanjaro Christian Medical University College Ethical Committee (CRERC) on behalf of the National Ethical Review Board of Tanzania and permission to conduct the study authorized by District Medical Officer (DMO) of Rufiji district.

\section{Results}

Table 1 summarizes demographic data of study participants. Most (58.9\%) of participants were young women aged between 20 and 35 years while those below 20 and above 35 years were $29.1 \%$ and $12 \%$ respectively. About two thirds $(66.3 \%)$ were married or cohabiting while $33.7 \%$ were single (unmarried, divorced or windowed). One third $(33.1 \%)$ of the participating mothers were pregnant for the first time (primigravidae) while 15.4\%, $13.1 \%$ and $12 \%$ were pregnant for the 2,3 and 4 times respectively. Those with $5^{\text {th }}$ pregnancy and above were $26.4 \%$, with $2.3 \%$ of these being pregnant for the $10^{\text {th }}$ time. Most of the mothers (63.4\%) had primary education, whereas only $7.4 \%$ had acquired secondary education and about one third (29.2\%) without any formal education. A large proportion of the mothers (97.7\%) used ITNs regularly and $52.5 \%$ of them took 2 doses of SP for IPTp while the rest either took one dose $(40.6 \%)$ or did not take SP at all $(6.9 \%)$. 
Table 1 - Socio-demographic and obstetric characteristics $(N=350)$

Characteristic

Group

Response

\% response

Socio-demographic characteristics

Maternal age

Marital status

Level of education

ITN's use
IPTp use

\section{Gravidity}

$$
\begin{gathered}
<20 \text { yrs } \\
20-35 \text { yrs } \\
>35 \text { yrs }
\end{gathered}
$$

Single

Married/cohabited

No formal education

Primary level

Secondary level
102

206

42

118

232

102

222

26
29.1

58.9

12

33.7

66.3

29.2

63.4

7.4

\section{Obstetrics characteristics}

Primigravida Multigravida

No SP dose Single dose Double dose
116

234

24

142

184

342

8
33.1

66.9

40.6

52.5

97.7

Abbreviation: IPTp = Intermittent Preventive Treatment for malaria in pregnancy; ITN = Insecticide Treated bed-Net

Factors associated with PM and prevalence of pregnancy outcomes

Among the 350 pregnant women studied, 28 (8\%; 95\% CI: $5.5-11.5 \%$ ) were found to have be positive for PM, while those who gave birth to newborns with low birth weight and those with maternal anemia and preterm birth were 22 (6.3\%; 95\% CI: 4.1-9.5\%), $160(59.3 \%$; 95\% CI: 53.1-65.2\%) and 26 (7.4\%; 95\% CI: 5-10.8\%) respectively. By using logistic regression, gravidity and history of fever during pregnancy were closely associ- ated with PM whereas maternal age, HIV status and ITN use were not. In terms of gravidity, multigravida were $70 \%$ less likely to have PM compared to primigravida (OR=0.3; CI $0.1-0.7)$. Pregnant women who had fever during their pregnancy were three times more likely to have PM compared to those without history of fever $(\mathrm{OR}=3.2$; CI $1.3-8.1)$. The study also showed that, maternal age $(\mathrm{OR}=1.0$; $\mathrm{CI} 0.4-1.9)$ and HIV status $(\mathrm{OR}=1.8$; CI 0.3 - 12.2) were neither associated with, nor were they risk factors for PM (Table 2). 
Table 2- Factors associated with placenta malaria

\begin{tabular}{|c|c|c|c|c|c|c|}
\hline \multirow[t]{2}{*}{ Characteristics } & \multicolumn{2}{|c|}{ Placenta malaria } & \multirow[t]{2}{*}{ Crude OR $(95 \% \mathrm{Cl})$} & \multirow[t]{2}{*}{ p-value } & \multirow[t]{2}{*}{ Adjusted OR (95\%Cl) } & \multirow[t]{2}{*}{$P$-value } \\
\hline & No & Yes & & & & \\
\hline \multicolumn{7}{|l|}{ Maternal age } \\
\hline$<25$ years & 172 & 16 & & & & \\
\hline$\geq 25$ years & 150 & 12 & $0.9(0.6-1.9)$ & 0.705 & $1.0(0.4-1.9)$ & 0.762 \\
\hline \multicolumn{7}{|l|}{ Gravidity } \\
\hline Primigravida & 100 & 16 & & & & \\
\hline Multigravida & 222 & 12 & $0.3(0.2-0.7)$ & 0.007 & $0.3(0.1-0.7)$ & 0.005 \\
\hline \multicolumn{7}{|l|}{ History of fever } \\
\hline No & 272 & 18 & & & & \\
\hline Yes & 50 & 10 & $3.0(1.3-6.9)$ & 0.009 & $3.2(1.3-8.1)$ & 0.013 \\
\hline \multicolumn{7}{|l|}{ HIV status } \\
\hline Sero-negative & 318 & 26 & & & & \\
\hline Sero-positive & 4 & 2 & $6.1(1.1-34.9)$ & 0.042 & $1.8(0.3-12.2)$ & 0.564 \\
\hline \multicolumn{7}{|l|}{ ITN use } \\
\hline No & 8 & 0 & & & & \\
\hline Yes & 314 & 28 & - & - & - & - \\
\hline
\end{tabular}

Abbreviation: P Malaria = Placenta malaria; OR = Odd ration; ITN = Insecticide Treated bed-Net

The study found that PM did not significantly predis- likely to develop maternal anemia (OR=3.4; CI 1.3 pose pregnant women to deliver babies with low birth 10.2) and four times more likely to have preterm birth weight $(\mathrm{OR}=2.4$; $\mathrm{CI} 0.7$ - 8.6). The study also revealed $\quad(\mathrm{OR}=3.8$; CI 1.3 - 11.4) compared to the ones without that, pregnant women with PM were three times more PM (Table 3).

TABLE 3 - Pregnancy outcomes based on status of placenta malaria

\begin{tabular}{|c|c|c|c|c|c|c|}
\hline \multirow[t]{2}{*}{ Birth outcome } & \multicolumn{2}{|c|}{$\begin{array}{l}\text { Placenta } \\
\text { malaria }\end{array}$} & \multirow[t]{2}{*}{$\begin{array}{c}\text { Crude OR } \\
(95 \% \mathrm{Cl})\end{array}$} & \multirow[t]{2}{*}{$\begin{array}{c}\text { p- } \\
\text { value }\end{array}$} & \multirow[t]{2}{*}{$\begin{array}{c}\text { Adjusted OR } \\
(95 \% \mathrm{Cl})\end{array}$} & \multirow[t]{2}{*}{$P$-value } \\
\hline & Yes & No & & & & \\
\hline \multicolumn{7}{|l|}{ Birth weight } \\
\hline$\geq 2.5 \mathrm{~kg}$ & 24 & 304 & & & & \\
\hline$<2.5 \mathrm{~kg}$ & 4 & 18 & $2.8(0.9-8.9)$ & 0.08 & $2.4(0.7-8.6)$ & 0.16 \\
\hline \multicolumn{7}{|c|}{ Maternal Hb-level* } \\
\hline$\geq 11 \mathrm{~g} / \mathrm{dl}$ & 6 & 104 & & & & \\
\hline$<11 \mathrm{~g} / \mathrm{dl}$ & 20 & 140 & $2.5(0.9-6.4)$ & 0.061 & $3.4(1.3-10.2)$ & 0.01 \\
\hline \multicolumn{7}{|l|}{ GA at birth } \\
\hline$\geq 37$ weeks & 22 & 302 & & & & \\
\hline$<37$ weeks & 6 & 20 & $4.1(1.5-11.3)$ & 0.006 & 3.8 (1.3-11.4) & 0.02 \\
\hline
\end{tabular}

Abbreviations: $\mathrm{GA}=$ Gestational age; $\mathrm{OR}=$ odds ratio; $\mathrm{Cl}=$ confidence interval; $\mathrm{Hb}=$ Haemoglobin level

*There were 80 participants with no $\mathrm{Hb}$ results

Number of IPTp-SP doses as a risk factor for PM The study showed that, use of 2 doses of SP during pregnancy did not significantly reduce the risk of developing $\mathrm{PM}(\mathrm{OR}=0.5$; CI $0.2-1.1)$, low birth weight
$(\mathrm{OR}=0.9 ; 0.3-2.1)$ and maternal anaemia $(\mathrm{OR}=1.1 ; \mathrm{CI}$ 0.7 - 1.8). However, 2 doses of SP was significantly associated with reduced risk of preterm birth to as much as ten times when compared to the ones who took single dose or none $(\mathrm{OR}=0.1$; CI $0.05-0.4)$ (Table 4) 
TABLE 4 - Effectiveness of IPTp-SP against placental malaria and adverse pregnancy outcome

\begin{tabular}{|c|c|c|c|c|c|c|}
\hline \multirow[t]{2}{*}{ Birth outcome } & \multicolumn{2}{|c|}{ IPTp-SP use } & \multirow[t]{2}{*}{ Crude OR $(95 \% \mathrm{Cl})$} & \multirow[t]{2}{*}{ p-value } & \multirow{2}{*}{$\begin{array}{l}\text { Adjusted OR } \\
(95 \% \mathrm{Cl})\end{array}$} & \multirow[t]{2}{*}{$P$-value } \\
\hline & $\begin{array}{c}<2 \\
\text { doses }\end{array}$ & $\begin{array}{c}2 \\
\text { doses }\end{array}$ & & & & \\
\hline \multicolumn{7}{|l|}{ Placental malaria } \\
\hline Negative & 148 & 174 & & & & \\
\hline Positive & 18 & 10 & $0.5(0.2-1.1)$ & 0.067 & $0.5(0.2-1.1)$ & 0.08 \\
\hline \multicolumn{7}{|l|}{ Birth weight } \\
\hline $2.5 \mathrm{~kg}$ and above & 154 & 174 & & & & \\
\hline$<2.5 \mathrm{~kg}$ & 12 & 10 & $0.7(0.3-1.8)$ & 0.491 & $0.9(0.3-2.1)$ & 0.73 \\
\hline \multicolumn{7}{|c|}{ Maternal Hb-level } \\
\hline$\geq 11 \mathrm{~g} / \mathrm{dl}$ & 60 & 50 & & & & \\
\hline$<11 \mathrm{~g} / \mathrm{dl}$ & 82 & 78 & $1.1(0.7-1.9)$ & 0.594 & $1.1(0.7-1.8)$ & 0.71 \\
\hline \multicolumn{7}{|l|}{ GA age at birth } \\
\hline$\geq 37$ weeks & 144 & 180 & & & & \\
\hline$<37$ weeks & 22 & 4 & $0.1(0.05-0.4)$ & 0.001 & $0.1(0.05-0.4)$ & $<0.001$ \\
\hline
\end{tabular}

Abbreviations: $\mathrm{GA}=$ Gestational age; $\mathrm{OR}=$ odds ratio; $\mathrm{Cl}=$ confidence interval; $\mathrm{Hb}=$ Haemoglobin level

*There were 80 participants with no $\mathrm{Hb}$ results

\section{Discussion}

Malaria in pregnancy is known to be a major public health problem that affects millions of women in tropical and subtropical regions. In many parts of Africa, the prevalence of PM is substantially high to about $25 \% 0^{9,21}$ Our data shows the prevalence of PM to be $28(8 \%)$ in the study site along the coast of Tanzania. The findings are similar to two previous studies that were conducted almost 7 years ago in two districts in Morogoro, Central Tanzania, areas within share similar malaria transmission intensity with Rufiji ${ }^{19,25}$. This may be an important concern of having a constant prevalence of PM for a period of time despite on-going malaria control interventions in the country.

We show in our study that primigravida were at higher risk of PM compared to multigravida. It is understood from previous studies that primigravida are at a higher risk of PM because of the absence of previous exposure to P.falciparum and therefore they have not yet developed protective antibodies that block the adhesion of parasitized erythrocytes to chondroitin sulfate A (CSA) in the placental intervillous space to promote clearance of parasites ${ }^{6,11}$. Protective immunity to P. falciparum malaria is therefore acquired following prolonged exposure in areas of endemic parasite transmission. Primi- gravidity as a risk factor for PM is a consensus among many studies that usually find it as an important risk factor for $\mathrm{PM}^{1,12,31}$. It is therefore important to prioritize malaria control measures among primigravida. History of fever was found to be a significant predictor of PM in this study. It is most likely in our case that, fever cases that were observed could have been secondary to previous malaria illness and/or PM. However, our study had limitations to differentiate between non malarial fever and confirmed malarial illness during pregnancy period. Fever during pregnancy has also been reported as an important predictor of PM in areas with stable malaria transmission $^{35}$.

We found in this study that the odds for women with PM to develop maternal anemia and to have preterm births were 3 and 4, respectively. Despite the absence of statistical significance for PM to predispose pregnant women to deliver babies with low birth weights, a weak predisposition of 2.4 times risk was seen. It has been explained in literatures that, parasitized erythrocytes in placenta induce local immunologic responses and complement activation that cause dysregulation of angiogenesis that leads to placental function insufficiency, fetal growth restriction and ultimately low birth weight ${ }^{6,36}$. PM in this study was found to predispose significantly 
pregnant women to maternal anemia $(\mathrm{P}=0.01)$ and preterm birth $(\mathrm{P}=0.02)$ but not low birth weight $(\mathrm{P}=0.16)$. There is a great extent of disparity between findings from different geographical regions. A study done in high malaria transmission areas of eastern Sudan which showed no significant association between PM and low birth weight (Adam et al. 2010). Study done in Accra Ghana by Ofori showed that, PM was significantly associated with low birth weight and maternal anemia ${ }^{30}$. In another study done by Bako and colleagues in North-Eastern Nigeria where malaria transmission is high, PM was significantly associated with low birth weight and cord parasitaemia ${ }^{3}$. Study done in Senegal by N'Dao and others showed PM was significantly associated with low birth weight, preterm birth and perinatal mortality ${ }^{26}$.

In malaria endemic countries like Tanzania, proportions of parasitized erythrocytes in pregnant women are usually higher in the placenta than in peripheral blood $18,24,32$ and with peripheral blood slides examination as the standard diagnostics, the real burden of malaria in pregnancy is almost always underestimated in these areas. Tanzanian National Malaria Control Program implements administration of at least two doses of SP in the second and third trimester as a standard regimen for controlling malaria in pregnancy. We show in our study however that, only half $(52.6 \%)$ of pregnant women took the recommended two doses of SP. A lesser proportion of $40.5 \%$ took a single dose whereas $6.9 \%$ never took any SP dose during pregnancy. According to reports from the National Bureau of Standards (NBS) and Tanzania Demographic and Health Survey (TDHS) in 2010, about one third of pregnant mothers (32.3\%) took at least two SP doses as recommended by the national policy. Our data show a notable increase of SP use for IPTp from 32.3\% in 2010 to $52.6 \%$ in 2012 of pregnant women. All these findings possess a major challenge to the newly recommended IPTp regimen that requires more than 3 doses of IPTp to be administered in pregnancy.

In order to determine the effectiveness of SP for IPTp, $\mathrm{PM}$ was considered the primary indicator since is determined solely by the presence of parasites in a non multi-factorial manner. However, secondarily, the effectiveness of SP for IPTp was also determined based on occurrence of adverse pregnancy outcome; low birth weight, maternal anemia and preterm birth. We show in this study that, pregnant women who took two doses of SP were not significantly protected against PM, LBW, MA. Preliminary data from previous studies have suggested a continued trend in reduction of effectiveness of SP for IPTp in many African countries ${ }^{13,37,39}$.

Our data supports the growing concern over the decreasing effectiveness of the two-dose regimen of SP for IPTp in high malaria transmission regions of East Africa ${ }^{17,33}$. With a great overlap between malaria and HIV incidences, the usefulness of SP for IPTp is not only currently questionable due to its widespread and increasing resistance, but also its interaction with HIV infections during pregnancy. The continued effectiveness of SP in such areas is therefore challenged by two parameters that must be addressed together if IPTp programs have to be useful.

With growing resistance of the parasite to SP, it is important that the regimen for SP use for IPTp is reviewed. Simply increasing the dose to 3 doses and above may require careful evaluation especially because SP is currently resisted by parasite strains isolated almost all over Tanzania. SP has been a relatively safe drug for use for IPTp, with the advantage of being taken once at a time thus maximizing compliance. The use of most other anti-malarial drugs for IP'Tp, e.g. ACTs, is challenged by their potential safety concern to fetus. At a point when the use of SP for IPTp is thus challenged, an integrated program that involves the use of alternative drugs, ITNs, IRS, and other strategies could be the most viable approach to controlling malaria during pregnancy.

\section{Conclusion}

The main result in our study was that, the use of two doses of SP, among pregnant women in Rufiji in Tanzania could not reduce the risk prevalence of PM as assessed by presence of parasitemia. Furthermore, we observed that SP use for IPTp did not have significantly direct benefit against PM, LBW, MA, although it was protective against pre term birth. Hence special consideration needs to be made when planning for malaria protective measures in pregnancy. The current SP regimen should be reviewed to accommodate the effect of increased resistance by parasites by either increasing the number of doses or devising and integrated interventional program that will include other effective malaria control approaches such as IRS and use of ITNs. Having a small sample size and limited to one study 
district are important study limitations of which the finding cannot easily be generalized in the Tanzanian population.

\section{Competing interests}

The authors declare that they have no competing interests.

\section{Authors' contributions}

$\mathrm{RN}$ and DM conceived the study and carried out data collection and analyses; AJ and JC contributed to the study design and data analysis; RN wrote the first draft; and JC and DM revised and developed the final submitted manuscript version. All authors contributed to the manuscript, read and approved the final submitted version.

\section{Acknowledgements}

The Author acknowledges the financial and logistical assistance provided by KCMUCo. The outstanding contribution of Alutu Masokoto of KCRI in microscopy is highly appreciated. Special thanks go to staff of Kibiti Health Centre for their support during data collection period and the pregnant mothers in Rufiji who participated in the study.

\section{References}

1. Albiti AH, Adam I, Ghouth AS. Placental malaria, anaemia and low birthweight in Yemen. Transactions of the Royal Society of Tropical Medicine and Hygiene. 2010;104:191-4.

2. Anders K, Marchant T, Chambo P, et al. Timing of intermittent preventive treatment for malaria during pregnancy and the implications of current policy on early uptake in north-east Tanzania. Malaria journal. 2008;7:79.

3. Bako BG, Audu BM, Geidam AD, et al. Prevalence, risk factors and effects of placental malaria in the UMTH, Maiduguri, North-eastern, Nigeria: a cross-sectional study. Journal of Obstetrics \& Gynecology. 2009;29:307-10.

4. Biswas S,Escalante A, Chaiyaroj S, et al. Prevalence of point mutations in the dihydrofolate reductase and dihydropteroate synthetase genes of Plasmodium falciparum isolates from India and Thailand: a molecular epidemiologic study. Trop Med Int Health. 2000;5:737-43. 5. Bray RS, Sinden RE. The sequestration of $<i>$ Plas- modium falciparum $</ i>$ infected erythrocytes in the placenta. Transactions of the Royal Society of Tropical Medicine and Hygiene. 1979;73:716-9.

6. Conroy AL, McDonald CR, Silver KL, et al. Complement activation: a critical mediator of adverse fetal outcomes in placental malaria? Trends in parasitology. 2011;27:294-9.

7. Dellicour S, Tatem AJ, Guerra CA, et al. Quantifying the number of pregnancies at risk of malaria in 2007: a demographic study. PLoS Med. 2010;7:e1000221.

8. Desai M, ter Kuile FO, Nosten F, et al. Epidemiology and burden of malaria in pregnancy. Lancet Infect Dis. 2007;7:93-104.

9. Desai M, ter Kuile FO, Nosten F, et al. Epidemiology and burden of malaria in pregnancy. Lancet Infect Dis. 2007;7:93-104.

10. Duffy MF, Byrne TJ, Elliott SR, et al. Broad analysis revealsa consistent pattern of var gene transcription in Plasmodium falciparum repeatedly selected for a defined adhesion phenotype. Molecular microbiology. 2005;56:774-88.

11. Elliott SR, Duffy MF, Byrne TJ, et al. Cross-reactive surface epitopes on chondroitin sulfate A-adherent Plasmodium falciparum-infected erythrocytes are associated with transcription of var2csa. Infection and immunity. 2005;73:2848-56.

12. Falade CO, Tongo OO, Ogunkunle OO, et al. Effects of malaria in pregnancy on newborn anthropometry. The Journal of Infection in Developing Countries. 2010;4:448-53.

13. Feng G, Simpson JA, Chaluluka E, et al. Decreasing burden of malaria in pregnancy in Malawian women and its relationship to use of intermittent preventive therapy or bed nets. PLoS One. 2010;5:e12012.

14. Gill CJ, Macleod WB, Mwanakasale V, et al. Inferiority of single-dose sulfadoxine-pyrimethamine intermittent preventive therapy for malaria during pregnancy among HIV-positive Zambian women. Journal of Infectious Diseases. 2007;196:1577-84.

15. Guyatt HL, Snow RW. Impact of malaria during pregnancy on low birth weight in sub-Saharan Africa. Clinical microbiology reviews. 2004;17:760-9.

16. Hamer DH, Mwanakasale V, MacLeod WB, et al. Two-dose versus monthly intermittent preventive treatment of malaria with sulfadoxine-pyrimethamine in HIV-seropositive pregnant Zambian women. Journal of Infectious Diseases. 2007;196:1585-94.

17. Harrington WE, Mutabingwa TK, Kabyemela E, et 
al. Intermittent treatment to prevent pregnancy malaria does not confer benefit in an area of widespread drug resistance. Clin Infect Dis. 2011;53:224-30.

18. Ibhanesebhor SE, Okolo AA. Placental malaria and pregnancy outcome. Int J Gynaecol Obstet. 1992;37:24752.

19. Kabanywanyi AM, MacArthur JR, Stolk WA, et al. Malaria in pregnant women in an area with sustained high coverage of insecticide-treated bed nets. Malar J. 2008;7:133.

20. Kalanda BF, van Buuren S, Verhoeff FH, et al. Catch-up growth in Malawian babies, a longitudinal study of normal and low birthweight babies born in a malarious endemic area. Early human development. 2005;81:841-50.

21. Kaur MR. Staff Nurses (Nicu) Knowledge Regarding Care Of Low Birth Weight Baby.

22. McGregor IA. Thoughts on malaria in pregnancy with consideration of some factors which influence remedial strategies. Parassitologia. 1987;29:153.

23. Meuris S, Piko BB, Eerens P, et al. Gestational malaria: assessment of its consequences on fetal growth. The American journal of tropical medicine and bygiene. 1993;48:603-9.

24. Mockenhaupt FP, Hamann L, von GC, et al. Common polymorphisms of toll-like receptors 4 and 9 are associated with the clinical manifestation of malaria during pregnancy. J Infect Dis. 2006;194:184-8.

25. Mosha TC, Ntarukimana D, John M. Prevalence of congenital malaria among neonates at morogoro regional hospital, morogoro. Tanzania. Tanzania J Health Res. 2010;12:1-10.

26. N'Dao CT, N'Diaye JL, Gaye A, et al. [Placental malaria and pregnancy outcome in a peri urban area in Senegal]. Revue d'epidemiologie et de sante publique. 2006;54:149-56.

27. Njau JD, Kabanywanyi AM, Goodman CA, et al. Adverse drug events resulting from use of drugs with sulphonamide-containing anti-malarials and artemisinin-based ingredients: findings on incidence and household costs from three districts with routine demographic surveillance systems in rural Tanzania. Malar J. 2013;12:236.

28. Njau JD, Stephenson R, Menon M, et al. Exploring the impact of targeted distribution of free bed nets on households bed net ownership, socio-economic disparities and childhood malaria infection rates: analysis of national malaria survey data from three sub-Saharan Africa countries. Malar J. 2013;12:245.

29. Nosten FTER, Ter Kuile F, Maelankirri L, et al. Malaria during pregnancy in an area of unstable endemicity. Transactions of the Royal Society of Tropical Medicine and Hygiene. 1991;85:424-9.

30. Ofori MF, Ansah E, Agyepong I, et al. Pregnancy-associated malaria in a rural community of Ghana. Ghana medical journal. 2009;43:13.

31. Ojurongbe O, Oyedeji SI, Oyibo WA, et al. Molecular surveillance of drug-resistant Plasmodium falciparum in two distinct geographical areas of Nigeria. Wiener klinische Wochenschrift. 2010;122:681-5.

32. Okoko BJ, Enwere G, Ota MO. The epidemiology and consequences of maternal malaria: a review of immunological basis. Acta Trop. 2003;87:193-205.

33. Sridaran S, McClintock SK, Syphard LM, et al. Anti-folate drug resistance in Africa: meta-analysis of reported dihydrofolate reductase (dhfr) and dihydropteroate synthase (dhps) mutant genotype frequencies in African Plasmodium falciparum parasite populations. Malar J. 2010;9:247.

34. Suguitan AL, Jr., Leke RG, Fouda G, et al. Changesin the levels of chemokines and cytokines in the placentas of women with Plasmodium falciparum malaria. J Infect Dis. 2003;188:1074-82.

35. Takem EN, D'Alessandro U. Malaria in pregnancy. Mediterr J Hematol Infect Dis. 2013;5:e2013010.

36. Uneke CJ. Impact of placental Plasmodium falciparum malaria on pregnancy and perinatal outcome in sub-Saharan Africa: II: effects of placental malaria on perinatal outcome; malaria and HIV. The Yale journal of biology and medicine. 2007;80:95.

37. van Eijk AM, Hill J, Alegana VA, et al. Coverage of malaria protection in pregnant women in sub-Saharan Africa: a synthesis and analysis of national survey data. Lancet Infect Dis. 2011;11:190-207.

38. WHO A. strategic framework for malaria prevention and control during pregnancy in the African region. World Health Organization, Brazzaville, The Republic of the Congo. 2004.

39. Wilson NO, Ceesay FK, Obed SA, et al. Intermittent preventive treatment with sulfadoxine-pyrimethamine against malaria and anemia in pregnant women. Am J Trop Med Hyg. 2011;85:12-21.

40. World Health Organization. The Africa malaria report 2003: World Health Organization Geneva; 2003. 